\title{
Compressed sensing of twisted photons
}

\author{
F. Bouchard ${ }^{1}$, D. Koutný ${ }^{2}$, F. Hufnagel ${ }^{1}$, Z. HRAdil ${ }^{2}$, J. ŘeháČEK ${ }^{2}$, \\ Y. S. TEO ${ }^{3}$, D. AHN ${ }^{3}$, H. JEONG ${ }^{3}$, L. L. SÁNCHEZ-SOTO ${ }^{4,5},^{*}$, G. LEUCHS ${ }^{1,5}$, AND \\ E. KARIMI 1,5
}

\author{
${ }^{1}$ Department of Physics, University of Ottawa, 25 Templeton Street, Ottawa, Ontario, K1N 6N5 Canada \\ ${ }^{2}$ Department of Optics, Palacký University, 17. listopadu 12, 77146 Olomouc, Czech Republic \\ ${ }^{3}$ Department of Physics and Astronomy, Seoul National University, 08826 Seoul, South Korea \\ ${ }^{4}$ Departamento de Óptica, Facultad de Física, Universidad Complutense, 28040 Madrid, Spain \\ ${ }^{5}$ Max-Planck-Institut für die Physik des Lichts, Staudtstraße 2, 91058 Erlangen, Germany \\ *Corresponding author: Isanchez@fis.ucm.es
}

The ability to completely characterize the state of a quantum system is an essential element for the emerging quantum technologies. Here, we present a compressed-sensing inspired method to ascertain any rank-deficient qudit state, which we experimentally encode in photonic orbital angular momentum. We efficiently reconstruct these qudit states from a few scans with an intensified CCD camera. Since it requires only a few intensity measurements, our technique would provide an easy and accurate way to identify quantum sources, channels, and systems. (C) 2016 Optical Society of America

OCIS codes: (270.0270) Quantum optics; (270.5585) Quantum information and processing; (120.3940) Metrology.

http://dx.doi.org/10.1364/optica.XX.XXXXXX

The orbital angular momentum (OAM) of single photons, which provides an unbounded vector space, has been recognized as a preeminent platform for encoding both quantum [1-3] and classical $[4,5]$ information. Although the generation of photons with OAM is relatively simple, the full characterization of a quantum state in the OAM Hilbert space stands as a challenging task. Several methods have demonstrated accurate projective measurements to determine OAM states [6-11]. However, these projective measurements work adequately only for pure states. The case of mixed states requires full state tomography, and this involves projective measurements on arbitrary superpositions of two or more OAM eigenstates [12], a task which remains challenging.

To circumvent these problems, we propose and experimentally demonstrate a method inspired by compressed sensing. Originating from the context of classical signal processing [13, 14], this technique harnesses prior assumptions about the state to reconstruct it from an undersampled set of measurements. It is routinely used to estimate vectors or matrices from incomplete information, with applications in many diverse fields of research $[15,17]$. Compressed sensing has also been adapted as a tool for state tomography of discrete systems in quantum theory [18-25]. Our scheme involves making a small number of intensity scans with an intensified CCD camera. The compressed sensing algorithms, supplemented with the positivity constraint [26], which operates as a kind of regularization, enables us to construct informationally complete measurements that are robust to noise and modeling errors. In other words, one can use a very simple setup for the characterization of a quantum state with a priori information about its nature.

We begin our analysis by recalling a few basic concepts that we will need to understand the technique. In any tomographic protocol, one infers the quantum state, represented by the density matrix $\rho$, from the distinct outcomes of a collection of measurements performed on identical copies of the system. The outcomes of these measurements are given by the Born rule $p_{\alpha}=\operatorname{Tr}\left(\rho \Pi_{\alpha}\right)$, where $\left\{\Pi_{\alpha}\right\}$ is the positive operator-valued measure (POVM) describing the setup [27]. We denote the action of the POVM by $\mathscr{A}: \rho \mapsto \mathbf{p}$, that maps $\rho$ onto the vector containing all the probabilities $\left\{p_{\alpha}\right\}$.

A POVM is informationally complete (IC) when the corresponding outcome probabilities are sufficient to determine an arbitrary state $[28,29]$. Obviously, the number of outcomes of any IC measurement is at least $d^{2}$, which makes traditional methods infeasible as the dimensionality of the system increases. However, if we know a priori that the rank of the system fulfills $\operatorname{rank}(\rho) \leq r$, with $r \ll d$, then we can substantially reduce the number of measurement samples required to uniquely reconstruct the unknown signal matrix. This can be accomplished with a map $\mathscr{A}$ that satisfies the appropriate properties [30]; the true state $\rho_{0}$ is then the only density matrix within the set of positive Hermitian matrices of any rank that yields the measurement probabilities $\mathbf{p}$ [26]. The corresponding estimator $\widehat{\rho}$ is given by,

$$
\widehat{\rho}=\min _{\rho}\|\mathscr{A}[\rho]-\mathbf{p}\| \quad \text { s.t. } \quad \rho \geq 0,
$$

whose solution can be efficiently found by convex programming [31].

The insights into the regularizing effect of the positivity constraint permit us to tackle reconstructing OAM quantum states. To represent the structure of the transverse field we will be using the well-known 
(a)

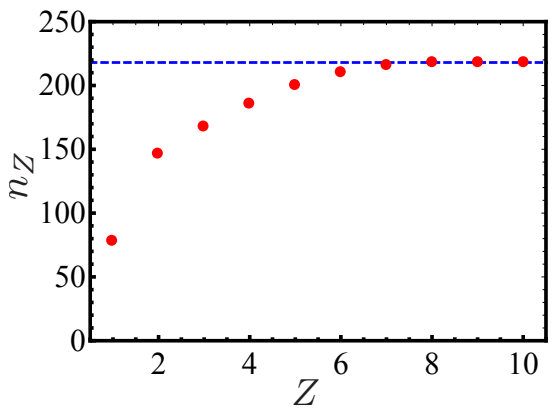

(b)

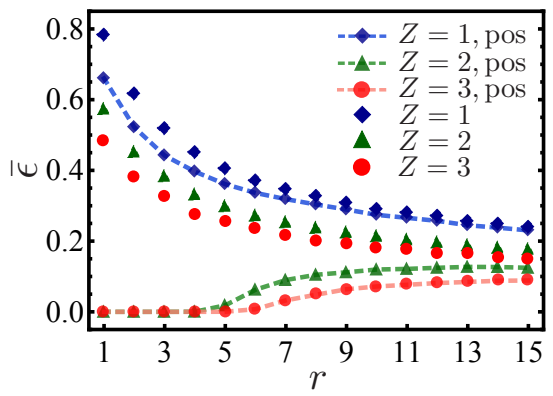

(c)

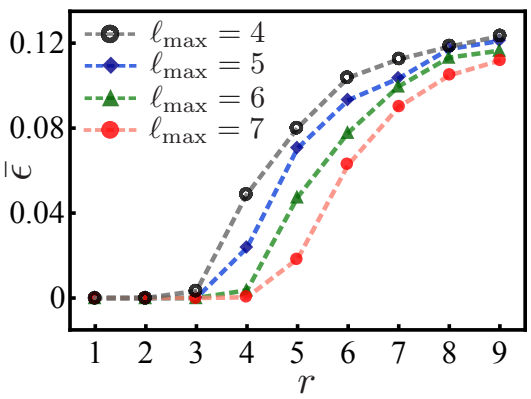

Fig. 1. Simulation of our compressed sensing protocol with twisted photons. (a) Number of independent detections $n_{Z}$ generated by $Z$ intensity scans performed on a signal with $\ell_{\max }=7(d=15)$. The maximum of $n_{Z}=218$ detections is obtained for $Z \geq 8$ scans. (b) Reconstructions errors, $\bar{\varepsilon}$, from the compressed sensing protocol of the twisted photons as a function of the rank of the state for a fixed dimension $\left(\ell_{\max }=7\right)$ and for $Z=1,2$, and 3 CCD scans, with and without positivity constraint. (c) The reconstructions errors for two CCD scans and different dimensions. In all the cases, we take a $19 \times 19$ pixels screen.

Laguerre-Gauss (LG) modes that can be written as [32],

$$
\begin{aligned}
& \operatorname{LG}_{p \ell}(r, \phi, z)=\langle r, \phi, z \mid \ell, p\rangle=\sqrt{\frac{2 p !}{\pi(p+|\ell|) !}} \frac{1}{w(z)}\left(\frac{\sqrt{2} r}{w(z)}\right)^{|\ell|} \\
& \times L_{p}^{|\ell|}\left(\frac{2 r^{2}}{w(z)^{2}}\right) \exp \left(-r^{2}\left[\frac{1}{w(z)^{2}}-i \frac{k}{2 R(z)}\right]-i \ell \phi-i \psi_{p \ell}(z)\right),
\end{aligned}
$$

where $(r, \phi, z)$ denote cylindrical coordinates, $k$ is the wave number, $L_{p}^{|\ell|}($.$) is the generalized Laguerre polynomial, \ell \in\{0, \pm 1, \pm 2, \ldots\}$ is the azimuthal mode index, and $p \in\{0,1,2, \ldots\}$ is the radial mode index, which is related to the number of radial nodes. The parameters $R(z), w(z)$, and $\Psi_{p \ell}(z)$ denote the radius curvature of the wave fronts, the beam radius, and the Gouy phase at the propagation distance $z$, respectively: $w^{2}(z)=w_{0}^{2}\left[1+\left(z / z_{\mathrm{R}}\right)^{2}\right], R(z)=z\left[1+\left(z_{\mathrm{R}} / z\right)^{2}\right]$, and $\psi_{p \ell}(z)=(2 p+|\ell|+1) \arctan \left(z / z_{R}\right)$, with the Rayleigh range $z_{\mathrm{R}}=$ $k w_{0}^{2} / 2$ and $w_{0}$ the beam waist, which we assume located at $z=0$. In what follows, we will set $p=0$ and denote $\psi_{\ell}(z)=\psi_{p=0 \ell}(z)$.

As mentioned before, we propose to reconstruct the signal in the LG basis from $Z$ intensity scans registered by a CCD camera positioned at distances $z_{1}, z_{2}, \ldots$, with respect to the beam waist, which is in the spirit of the Gerchberg-Saxton algorithm [33]. This amounts to projecting the density matrix on free-space position eigenstates; viz,

$$
p(r, \phi, z)=\langle r, \phi, z|\rho| r, \phi, z\rangle \propto e^{-2 \mathfrak{r}^{2}} \sum_{\ell \ell^{\prime}} \rho_{\ell \ell^{\prime}} C_{\ell \ell^{\prime}},
$$

where $C_{\ell \ell^{\prime}}=\mathfrak{r}^{|\ell|+\left|\ell^{\prime}\right|} \exp \left[i\left(\ell-\ell^{\prime}\right) \phi\right] \exp \left\{i\left[\psi_{\ell}(z)-\psi_{\ell^{\prime}}(z)\right]\right\}$ and $\mathfrak{r}(z)=$ $r / w(z)$. Each combination of $r, \phi$, and $z$ coordinates, which corresponds to the associated pixel readings, gives us one particular linear combination of density matrix elements. Evidently by varying $r, \phi$, and $z$, the more linearly independent these generated combinations are, the more IC the POVM is.

Let us first consider $d$-dimensional states that can be represented in the LG mode basis with nonnegative azimuthal indices only; i.e., $\ell \in\{0,1, \ldots, d-1\}$. Incompleteness arises whenever two different pairs $\left(\ell^{\prime}, \ell^{\prime \prime}\right)$ generate the same $C_{\ell^{\prime} \ell^{\prime \prime}}$ for all $r$ and $\phi$. Surprisingly, it turns out (see Supplemental Material) that all density matrix elements can be uniquely determined from a single CCD image for any dimension $d$. Put differently, a simple von Neumann measurement (CCD scan) defined in an infinitely large space, becomes an IC POVM when projected into a finite space.

Next, we examine the case in which the sign of the topological charge is not constrained; that is, $d=2 \ell_{\max }+1$, with $\ell \in$ $\left\{-\ell_{\max }, \cdots, 0, \cdots, \ell_{\max }\right\}$. CCD scans are no longer IC, no matter how many planes $Z$ are sampled. As discussed in the Supplemental Material, the maximum number of linearly independent measurements generated by CCD scans is $n_{Z \rightarrow \infty}=d^{2}-(d-1) / 2$. Only for large dimensions, $d^{2} \gg 1$, does the measurement become nearly IC. The first two scans generate $n_{Z=1}=\left(d^{2}+6 d-3\right) / 4$ and $n_{Z=2}=\left(d^{2}+5 d-8\right) / 2$ independent detections. For higher $Z$, the new independent detections grow linearly with $\ell_{\max }$. A typical behaviour is shown in Fig. 1a) for $\ell_{\max }=7$ : the maximum $n_{Z \rightarrow \infty}$ is approximately attained with 8 scans. Actually, one can determine (see Supplemental Material) that the minimum scans required to approximately attain the maximum number of independent detections in the signal space is $Z_{\min }=\ell_{\max }+1$. Roughly one quarter and one half of this total is already available after one and two scans, respectively.

The compressive tomography is done by solving Eq. (1), where now $\alpha=\{r, \phi, z\}$, and $\Pi_{\alpha}$ is defined by $\operatorname{Tr}\left(\rho \Pi_{\alpha}\right)=p(r, \phi, z)$. We first perform numerical simulations to explore the method. A set of random states of a given rank in the $\ell_{\max }=7$ space is chosen and scanning measurements are simulated for $Z=1,2$, and 3 CCD planes with a $19 \times 19$ pixel geometry.

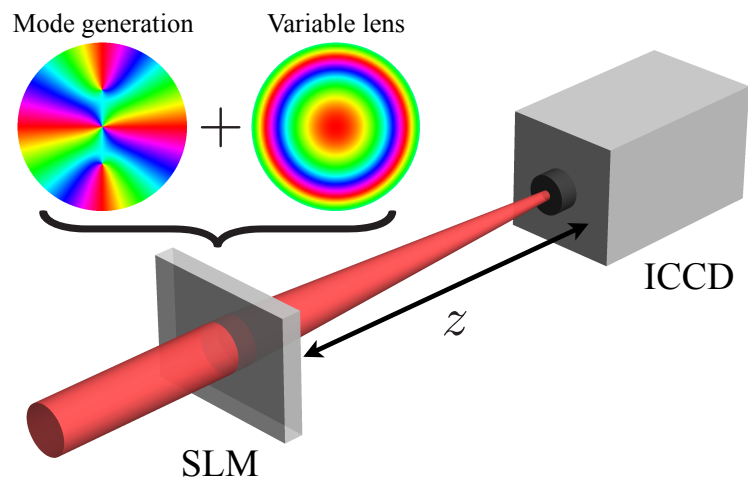

Fig. 2. Sketch of the experimental setup. A photonic state is generated by manipulating the phase and intensity of an incoming beam via the spatial light modulator (SLM). The beam is then focused using a variable holographic lens imprinted on the SLM together with the state generation hologram. The ICCD camera has a fixed position and records intensity scans. Inset shows the state and lens phase patterns, $[0,2 \pi)$, in a hue color. 
(a)

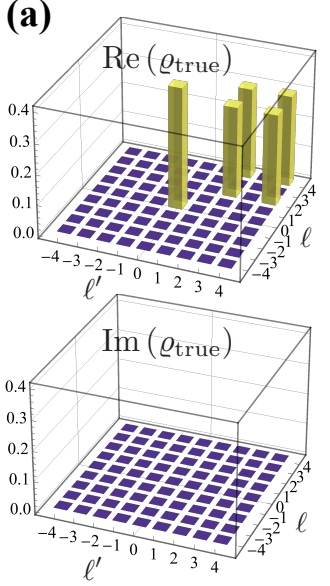

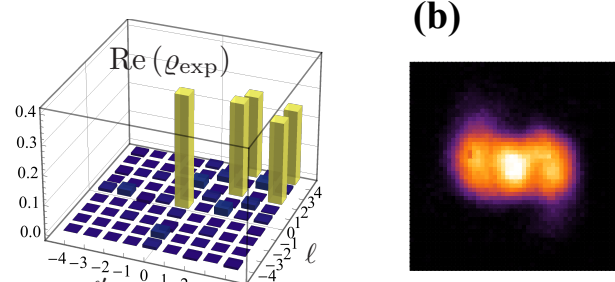
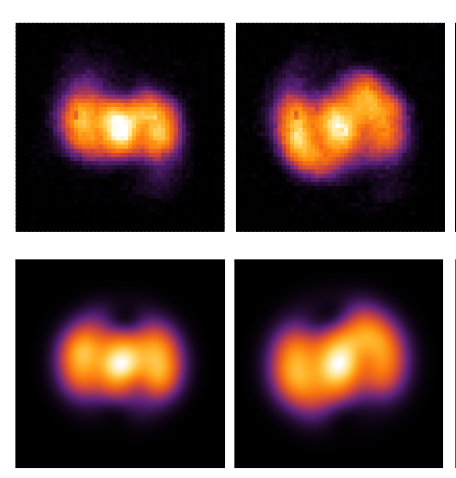
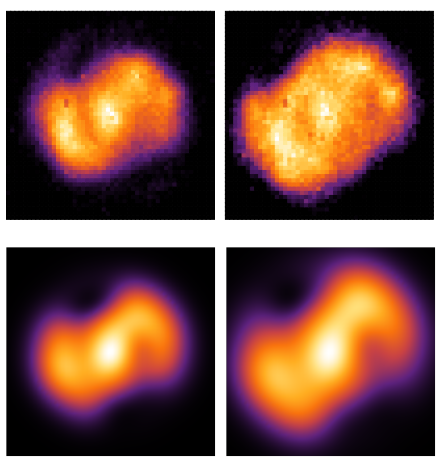

Fig. 3. Experimental reconstruction from compressed sensing. (a) Density matrix of the true state and (b) Reconstructed density matrix after two intensity scans with a signal space spanned by $\ell \in\{-4, \ldots, 4\}$. The upper row shows four experimental ICCD scans at the planes $z / z_{\mathrm{R}}=0,1 / 3$, $1 / 2$, and 1 , respectively. The lower row shows the predictions from the reconstructed state of the same ICCD scans at before.

The data is subject to quantum state reconstruction with and without the positivity constraints, where the latter is implemented with the help of the Moore-Penrose pseudoinverse [34]. Finally, for each true state, the corresponding Hilbert-Schmidt distances between the true and reconstructed states are calculated and the mean is taken over the set of true states of the same rank. We take this as the error $\bar{\varepsilon}=\mathbb{E}\left[\operatorname{Tr}\left(\rho_{\text {true }}-\widehat{\rho}\right)^{2}\right]$.

In Fig. 1b), we have plotted the errors $\bar{\varepsilon}$ as a function of the rank of the state, for $\ell_{\max }=7$. We observe a strong regularizing effect of the positivity constraint, especially on low-rank signals. First, positivity always makes the reconstruction errors smaller. Second, it cures the informational incompleteness of the tomography, provided the complexity of the measured signal stays below a certain threshold. Last, this threshold gets increased as we collect more scans. Notice though that protocols with positivity perform worse on more complex signals because the regularizing effect of positivity weakens. Loosely speaking, in comparing neighbourhoods of low- and high-rank states, one finds more nonphysical matrices in the former. Those objects get filtered by the positivity constraints leading to improved performance. The opposite is true of the protocols without positivity. Here, the more mixed states are less biased in the space of density matrices, so their average distance to the reconstructed matrix is smaller. Notice that this discussion does not apply to mutually compatible observations, for which positivity constraints are of no consequence. This can be observed in Fig. 1c) for a single scan, where the regularizing effect of positivity disappears. Stronger incompatibilities introduced by detections from different planes $\left[\Pi_{\alpha}, \Pi_{\alpha^{\prime}}\right] \neq 0$ are required to promote signal sparsity. The influence of the signal dimension with a fixed measurement is also illustrated in Fig. 1c), for the particular case of two scans. The quality of the compressive OAM tomography improves with the signal dimension. At first glance, this might seem counterintuitive; however, note that the sparsity of a signal of a given rank grows with dimension and so do the regularizing effects of positivity constraints.

To verify our scheme, we construct an experimental setup that allows us to generate and detect vortex beams at the single photon level using a spatial light modulator (SLM) and an intensified CCD (ICCD) camera, respectively. A simplified sketch is shown in Fig. 2. A quasicontinuous UV laser, at a wavelength of $355 \mathrm{~nm}$, is used to pump a type-I $\beta$-barium borate (BBO) crystal in order to generate single photons via spontaneous parametric downconversion. The spatial modes of the generated photons are filtered to the fundamental Gaussian mode by coupling them to a single mode fibre (SMF). The photons are then coupled out of the SMF and are made incident on an SLM, (X10468-07, Hamamatsu) consisting of an electronically controlled nematic liquid crystal device with $792 \times 600$ pixels. The phase and the intensity of the generated photons are controlled via a holographic intensity masking technique [35], with a diffraction efficiency greater than $70 \%$. To achieve a higher mode quality, a $10 \times$ microscope objective with a numerical aperture of 0.25 is employed to obtain a relatively large collimated beam at the SLM. The mode waist of the amplitude-modulated hologram at the SLM is determined to have an effectively flat phase and intensity for the incoming beam over the region of interest of the hologram. By doing so, we may generate any arbitrary spatial mode with a high-level of accuracy. In the case of pure states, a single hologram is used for shaping the transverse modes of all photons in the ensemble. For mixed states, the appropriate holograms are randomly varied to generate incoherent statistical mixtures of the desired modes. A single intensity scan is then recorded over the whole ensemble of generated photons.

To observe the intensity variation of LG modes upon propagation, due to the differences in Gouy phases, the beam must propagate for distances that are on the order of the Rayleigh range $z_{\mathrm{R}}$. This is achieved by simply focusing the beam. In the scheme described above, the ICCD camera is moved along the path of the beam in the $z$-direction. However, this is equivalent to varying the focal length of the lens and considering a fixed position of the ICCD camera. This is accomplished by imprinting a phase profile of the form $\exp \left(-i k r^{2} / 2 f\right)$, where $f$ is the focal length of the flat lens. By doing so, our experimental reconstruction does not require any mechanical displacement of the components in the setup. Intensity scans are recorded for different focal lengths corresponding to positions of $z / z_{\mathrm{R}}=0,1 / 3,1 / 2$ and 1 .

The true states are chosen to be following rank-2 states,

$$
\rho=p|0\rangle\langle 0|+(1-p)| \Psi\rangle\langle\Psi|,
$$

with $|\Psi\rangle=\cos \theta|-3\rangle+\sin \theta|3\rangle$. In the experiment, we randomly generate 20 states for values of $p$ and $\theta$ chosen. The experiment was also carried out with other sets of states; the results can be found in the Supplemental Material. In Fig. 3, we present the results for a typical state $\rho$ with two ICCD scans. The corresponding reconstructed density matrix is plotted in the left column. Even though we are dealing with informationally incomplete measurements, the protocol with positivity using two intensity scans fits the theoretical data quite well. Without the 


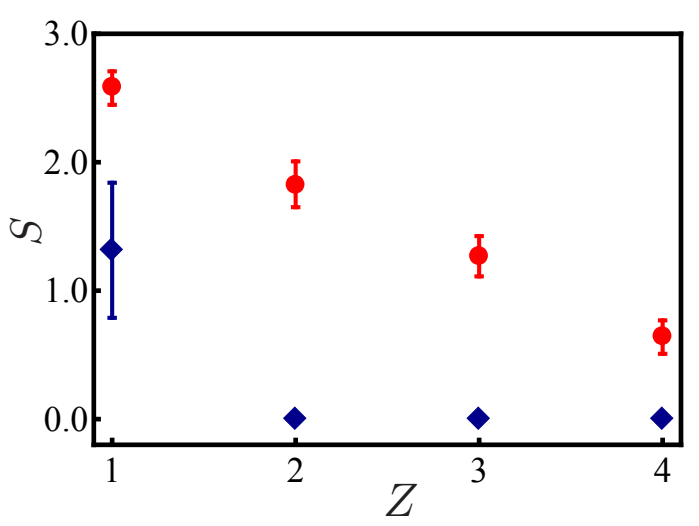

Fig. 4. Entropy $S$ as a function of the number of intensity scans $Z$, for a signal space spanned by $\ell \in\{-4, \ldots, 4\}$ and for true states of the form in Eq. (4). The circles are obtained by averaging over 20 random states and the error bars indicate the corresponding variance. Blue refers to protocol with positivity, while red is without positivity.

positivity constraint, the reconstructed states are very different from the true ones. We have also experimentally checked that when the signal space is constrained to only positive topological charges $\ell$, one intensity scan is enough to accurately predict the intensity scans in other planes, as anticipated by our theory. To assess the degree of IC for the intensity scans, we first consider a matrix of reconstructed states $\left\{\widehat{\rho}_{j}\right\}$ with its $j$ th column defined as a flattened $\rho_{j}$. A unique solution for the extremal problem (1) then implies that the entropy of normalized singular values for this matrix is zero. To compute the entropy, we reconstructed 20 true states for every ICCD scan, and average over all of them. As can be seen in Fig. 4, the protocol with positivity constraints is enough to characterize the input state with solely two scans. We corroborate once again that if we restrict our space to be spanned only with nonnegative azimuthal indices, the measurement is IC for both strategies (with and without positivity) with only one intensity scan.

In summary, we have developed a compressed sensing scheme able to uniquely reconstruct any rank-deficient qudit state encoded in the OAM degree of freedom. The positivity constraint has played a substantial role as a powerful regularization to perform a tomographic reconstruction in the regime of informationally incomplete data for intermediately sized quantum systems. This establishes a novel and efficient tomographic paradigm for OAM systems that could trigger interesting experimental research on complex quantum states, which otherwise might have been infeasible with currently known detection schemes in such experiments.

This work was supported by the European Union's Horizon 2020 Research and Innovation Programme (Q-SORT) grant number 766970, Canada Research Chairs (CRC), the BK21 Plus Program (21A20131111123) funded by the Ministry of Education (MOE, Korea) and National Research Foundation of Korea (NRF), the NRF grant funded by the Korea government (MSIP) (Grant No. 20100018295), the Korea Institute of Science and Technology Institutional Program (Project No. 2E27800-18-P043), the Spanish MINECO (Grant FIS2015-67963-P), the Grant Agency of the Czech Republic (Grant No. 18-04291S), and the IGA Project of the Palacký University (Grant No. IGA PrF 2018-003).

\section{REFERENCES}

1. G. Vallone, V. D’Ambrosio, A. Sponselli, S. Slussarenko, L. Marrucci, F. Sciarrino, and P. Villoresi, Phys. Rev. Lett. 113, 060503 (2014).
2. M. Mirhosseini, O. S. Magaña-Loaiza, M. N. O'Sullivan, B. Rodenburg, M. Malik, M. P. J. Lavery, M. J. Padgett, D. J. Gauthier, and R. W. Boyd, New J. Phys. 17, 033033 (2015).

3. A. Sit, F. Bouchard, R. Fickler, J. Gagnon-Bischoff, H. Larocque, K. Heshami, D. Elser, C. Peuntinger, K. Günthner, B. Heim, C. Marquardt, G. Leuchs, R. W. Boyd, and E. Karimi, Optica 4, 1006 (2017).

4. J. Wang, J.-Y. Yang, I. M. Fazal, N. Ahmed, Y. Yan, H. Huang, Y. Ren, Y. Yue, S. Dolinar, M. Tur, and A. E. Willner, Nat. Photonics 6, 488 EP (2012).

5. G. Gibson, J. Courtial, M. J. Padgett, M. Vasnetsov, V. Pas'ko, S. M. Barnett, and S. Franke-Arnold, Opt. Express 12, 5448 (2004).

6. A. Mair, A. Vaziri, G. Weihs, and A. Zeilinger, Nature 412, 313 (2001).

7. J. Leach, M. J. Padgett, S. M. Barnett, S. Franke-Arnold, and J. Courtial, Phys. Rev. Lett. 88, 257901 (2002).

8. J. Leach, J. Courtial, K. Skeldon, S. M. Barnett, S. Franke-Arnold, and M. J. Padgett, Phys. Rev. Lett. 92, 013601 (2004).

9. E. Karimi, B. Piccirillo, E. Nagali, L. Marrucci, and E. Santamato, Appl. Phys. Lett. 94, 231124 (2009).

10. G. C. G. Berkhout, M. P. J. Lavery, J. Courtial, M. Beijersbergen, and M. J. Padgett, Phys. Rev. Lett. 105, 153601 (2010).

11. M. Mirhosseini, M. Malik, Z. Shi, and R. W. Boyd, Nat. Commun. 4, 2781 (2013).

12. N. Bent, H. Qassim, A. A. Tahir, D. Sych, G. Leuchs, L. L. Sánchez-Soto, E. Karimi, and R. W. Boyd, Phys. Rev. X 5, 041006 (2015).

13. E. J. Candes, J. Romberg, and T. Tao, IEEE Trans. Inform. Theory 52, 489 (2006).

14. E. J. Candes and T. Tao, IEEE Trans. Inform. Theory 56, 2053 (2010).

15. Y. C. Eldar and G. Kutyniok, Compressed Sensing: Theory and Applications (Cambridge University Press, 2012).

16. S. Foucart and H. A. Rauhut, Mathematical Introduction to Compressive Sensing (Springer, 2013).

17. A. Stern, Optical Compressive Imaging (CRC Press, Boca Raton, 2016).

18. D. Gross, Y.-K. Liu, S. T. Flammia, S. Becker, and J. Eisert, Phys. Rev. Lett. 105, 150401 (2010).

19. A. Shabani, R. L. Kosut, M. Mohseni, H. Rabitz, M. A. Broome, M. P. Almeida, A. Fedrizzi, and A. G. White, Phys. Rev. Lett. 106, 100401 (2011).

20. W.-T. Liu, T. Zhang, J.-Y. Liu, P.-X. Chen, and J.-M. Yuan, Phys. Rev. Lett. 108, 170403 (2012).

21. C. Schwemmer, G. Tóth, A. Niggebaum, T. Moroder, D. Gross, O. Gühne, and H. Weinfurter, Phys. Rev. Lett. 113, 040503 (2014).

22. A. V. Rodionov, A. Veitia, R. Barends, J. Kelly, D. Sank, J. Wenner, J. M. Martinis, R. L. Kosut, and A. N. Korotkov, Phys. Rev. B 90, 144504 (2014).

23. F. Tonolini, S. Chan, M. Agnew, A. Lindsay, and J. Leach, Sci. Rep. 4, 6542 EP (2014).

24. A. Steffens, C. A. Riofrío, W. McCutcheon, I. Roth, B. A. Bell, A. McMillan, M. S. Tame, J. G. Rarity, and J. Eisert, Quantum Sci. Technol. 2, 025005 (2017).

25. C. A. Riofrío, D. Gross, S. T. Flammia, T. Monz, D. Nigg, R. Blatt, and J. Eisert, Nat. Commun. 8, 15305 (2017).

26. A. Kalev, R. L. Kosut, and I. H. Deutsch, npj quantum inf. 1, 15018 (2015).

27. A. Peres, Quantum Theory: Concepts and Methods (Kluwer, 2002).

28. E. Prugovečki, Int. J. Theor. Phys. 16, 321 (1977).

29. P. Busch and P. J. Lahti, Found. Phys. 19, 633 (1989).

30. E. J. Candès, C. R. Math. 346, 589 (2008). 
31. S. Boyd and L. Vandenberghe, Convex Optimization (Cambridge University Press, 2004).

32. A. Siegman, Lasers (Oxford University Press, 1986).

33. R. W. Gerchberg and W. O. Saxton, Optik 35, 237 (1972).

34. A. Ben-Israel and T. N. E. Greville, Generalized Inverses: Theory and Applications (Wiley, 1977).

35. E. Bolduc, N. Bent, E. Santamato, E. Karimi, and R. W. Boyd, Opt. Lett. 38, 3546 (2013). 